\title{
Modeling and Analysis of an Up-conversion Electret Electrostatic Energy Harvester
}

\author{
Shao-Hua NIU ${ }^{a^{*}}$, Shi-Qiao GAO ${ }^{b}$, and Chun-Hui GAO
}

School of Microelectronics, Beijing Institute of Technology, 5 South Zhongguancun Street, Haidian District, Beijing, China

ashh@bit.edu.cn, bgaoshq@bit.edu.cn, cdzjdchunhui@126.com

Keywords: Electret, Electrostatic Energy Harvester, Up-conversion.

\begin{abstract}
In order to reduce the environmental sensitive frequency of electret-based vibration electrostatic energy harvester (E-VEH), an up-conversion structure is designed. This harvester is a double cantilever structure includes a low frequency beam and high frequency beam. Electret is bounded to the high frequency beam, the low frequency beam vibrates excited by environment, and impacts the high frequency beam. The model of this structure is established and analyzed firstly. Then a prototype is made and tested. According to the measurement result, this kind of structure can convert part of the vibration energy within the vibration frequency between $1 \mathrm{~Hz}$ and $70 \mathrm{~Hz}$ into electrical energy under the action of the excitation load with the amplitude is $8 \mathrm{~m} / \mathrm{s}^{2}$. This indicates that the up-conversion structure can validly reduce the sense frequency of E-VEH, and can expand the harvester work bands.
\end{abstract}

\section{Introduction}

With the rapid development of MEMS, COMS sensors and data processing, the times of internet of things is coming. As an important part of internet of things, wireless sensor networks are rapidly growing. At present, chemical batteries are used for suppling power to wireless sensor networks. However, it hampers the development of wireless sensor networks because chemical batteries have maintenance, environmental and size issues. In recent years, it is concerned and studied how to harvest environment energy and convert it into electric power [1-3]. The vibration harvester has been paid more attention because vibration happens in anywhere and anytime. For vibration harvester, three mechanisms have been proposed: piezoelectric, electromagnetic and electrostatic Compared with piezoelectric and electromagnetic energy harvesters, electrostatic systems have advantages of compatibility with MEMS processes and small size. Electret-based vibration energy harvesters (E-VEHs) can be divided into two types, in-plane and out-of-plane. Compared with the in-plan E-VEHs, Out-of-plane E-VEHs have lower production costs because the device structure and process are relatively simple. Driven by the merit of low production costs, more and more researchers have focused on out-of-plane E-VEHs and attempted to improve their performance. When designing structure of out-of-plane E-VEHs, cantilever or double-end fixed beam is commonly employed. The size of these harvesters is usually small as their employers are always small. Therefor the natural frequency of most harvester structures is relatively high, always at the level of KHz. However, in natural environment, vibration frequency is always at the level of $\mathrm{Hz}$. In order to increasing the harvest efficiency, it is an important point to decrease the structure natural frequency.

In this paper, an up-conversion structure is introduced to reduce the environmental sensitive frequency of the E-VEHs. The model of this structure is established, and its characteristics are analyzed. By test, this up-conversion structure can effectively decrease the sensitive frequency of cantilever E-VEHS.

\section{Work Mechanism of E-VEHs}

The core of E-VEHs is a kind of dielectric function material called electret which can keep space charge or dipole charge for a long time. With the space electret method promotion, more and more space charge electret, as shown in Fig.1 (a), has been used, especially for vibration energy harvester. 
When it is used for harvest energy unit, its work mechanism can be described as Fig.1 (b). The unit is composed of electret, electrode and counter-electrode. According to Gauss's law, the amount of charges on electret $Q_{e}$ is equal to the sum of $Q_{b}$ and $Q_{t}$, where $Q_{b}$ is the total amount of charges on the electrode and $Q_{t}$ is the total amount of charges on the counter-electrode, i.e. $Q_{e}=Q_{b}+Q_{t}$. When the distance between the electrode and the counter-electrode is changed due to external excitation, the capacitance between counter-electrode and electret will change, and charges on electrode and counter-electrode will redistribute, which will induce current in the circuit. So the unit can be equivalent to a model as shown in Fig.1(c), which can be described as Eq.1. The surface potential $V_{s}$ could be considered as a monotonous linear function of the initial charges $Q_{e}$ of electret, $V_{s}=Q_{e} / C_{2}$.

$$
\frac{d Q_{t}(t)}{d t}=\frac{V_{s}}{R}-\frac{Q_{t}(t)}{R C(t)}
$$

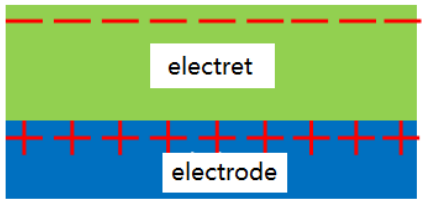

(a) Schematic of electret

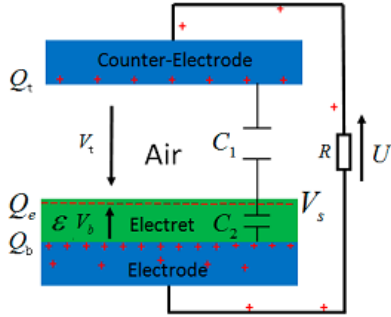

(b) Charge circulation

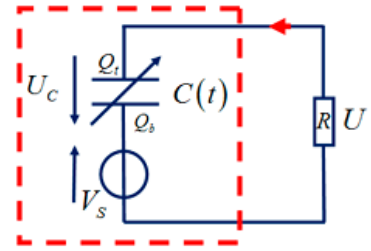

(c) Equivalent circuit model

Fig. 1 The mechanism of E-VEHs

\section{Up-conversion of E-VEHs with Double Cantilever}

According to the mechanism of E-VEHs, its output is related to vibration amplitude of the harvester structure, greater amplitude and higher output voltage. For present small-scale harvester, its harvest energy efficiency is relatively low, because its natural frequency is high while the vibration frequency of environment is low. To reduce the sense frequency of E-VEHs, an up-conversion structure with double cantilever is adopted.

\section{Model of the Up-conversion E-VEHs}

The up-conversion E-VEHs is composed of low frequency beam (LFB), high frequency beam (HFB), electret, as shown in Fig.2. At the free end of low frequency beam, a mass is fixed to decrease its natural frequency. Counter-electrode is laid on the high frequency beam. The electret and electrode is laid on the base. When external low frequency excitation acts on the base, the LFB will vibrate at relative high amplitude due to its low natural frequency. During the vibration of LFB, it will impact on the HFB. Thus the two beam will move together in the direction of electret, and the gap between HFB and electret will be changed, so that there will be current in circuit when loads are connected to the counter-electrode and electrode. Thus the harvester structure sense low frequency excitation.

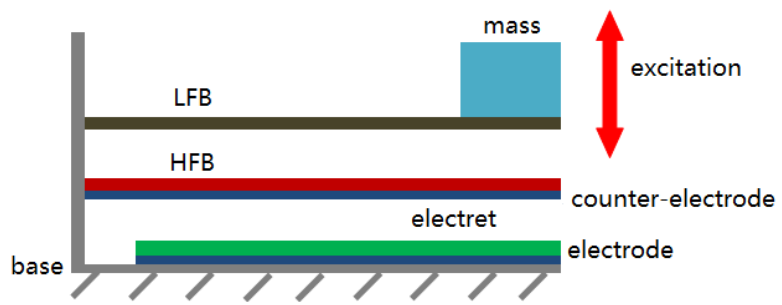

Fig. 2 Schematic of up-conversion E-VEHs 
According to the work principle mentioned above, the up-conversion structure can be equivalent to the model as shown in Fig.3. There $k_{1}$ and $k_{2}$ denote the stiffness of LFB and HFB respectively, $c_{1}$ and $c_{2}$ denote the damping coefficient of LFB and HFB respectively. So its dynamic characteristics can be written as

$$
x^{\prime \prime}+p_{1}\left(x, x^{\prime}\right)=f_{1}\left(y, y^{\prime}\right)
$$

Where

$$
\begin{gathered}
p_{1}\left(x, x^{\prime}\right)=\left\{\begin{array}{cc}
2 \xi_{3} \omega_{3} x^{\prime}+\omega_{3}^{2} x-\omega_{2}^{2} \Delta & x-y>\Delta \\
2 \xi_{1} \omega_{1} x^{\prime}+\omega_{1}^{2} x & x-y<\Delta
\end{array}\right. \\
f_{1}\left(x, x^{\prime}\right)=\left\{\begin{array}{cc}
2 \xi_{3} \omega_{3} y^{\prime}+\omega_{3}^{2} y & x-y>\Delta \\
2 \xi_{1} \omega_{1} y^{\prime}+\omega_{1}^{2} y & x-y<\Delta
\end{array}\right.
\end{gathered}
$$

In equation (3) and (4), there

$$
\begin{aligned}
& 2 \xi_{1} \omega_{1}=\frac{c_{1}}{m}, 2 \xi_{2} \omega_{2}=\frac{c_{2}}{m} 2 \xi_{3} \omega_{3}=\frac{c_{1}+c_{2}}{m}=\frac{c_{3}}{m}=2 \xi_{1} \omega_{1}+2 \xi_{2} \omega_{2} \omega_{1}^{2}=\frac{k_{1}}{m}, \omega_{2}^{2}=\frac{k_{2}}{m}, \\
& \omega_{3}^{2}=\frac{k_{1}+k_{2}}{m}=\frac{k_{3}}{m}=\omega_{1}^{2}+\omega_{2}^{2}
\end{aligned}
$$

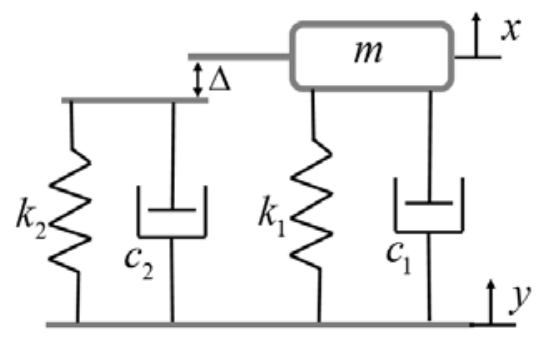

Fig. 3 Model of up-conversion structure

When the LFB impacts on the free end of HFB, i.e. the $\Delta=0$ in Fig.3, the HFB will vibrate and its displacement can be written as

$$
z(t)=\left\{\begin{array}{cc}
z_{M} e^{-\xi_{3} \omega_{3} t} \sin \left(\omega_{3} t\right) & \Rightarrow n \frac{2 \pi}{\omega} \leq t \leq(n+1) \frac{3 \pi}{2 \omega_{3}} \\
z_{M} e^{-\xi_{2} \omega_{2} t} \sin \left(\omega_{2}\left(t-(n+1) \frac{3 \pi}{2 \omega_{3}}\right)+\frac{3 \pi}{2}\right) & \Rightarrow(n+1) \frac{3 \pi}{2 \omega_{3}} \leq t \leq(n+1) \frac{2 \pi}{\omega} \\
z_{M}=a_{M}-\Delta &
\end{array}\right.
$$

Where $a_{M}$ is the vibration amplitude of LFB free end, $\Delta$ is the gap between LFB and HFB, $z_{M}$ is the amplitude of HFB free end, $n=0,1,2,3, \cdots$ is impact times of the two beams, $\xi_{3}$ is the couple damping ratio, $\xi_{2}$ is the damping ratio of HFB, $\omega$ is the frequency of excitation acceleration, $\omega_{2}$ is the natural frequency of HFB, $\omega_{3}$ is the couple natural frequency.

In Fig. 4 the vibration response of HFB is given under the excitation condition of $10 \mathrm{~m} / \mathrm{s}^{2}, 14 \mathrm{~Hz}$. It can be found that during an impact period the coupling vibration time is $3 \pi / 2 \omega_{3}$ which is less than the vibration period of LFB. After the LFB and HFB separate, the HFB will do damped free vibration until next impact comes. 


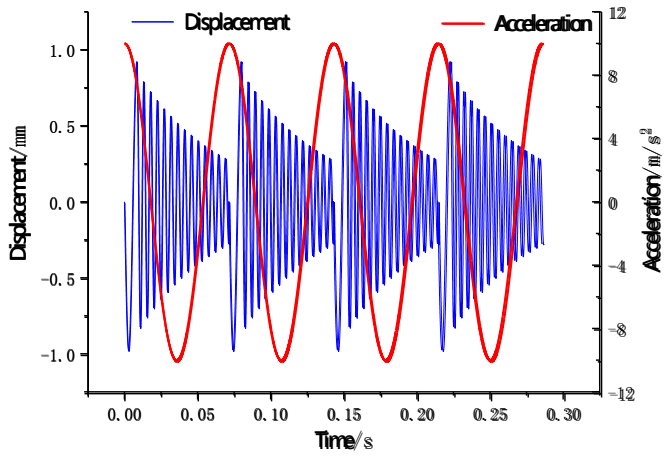

(a) vibration curve of HFB

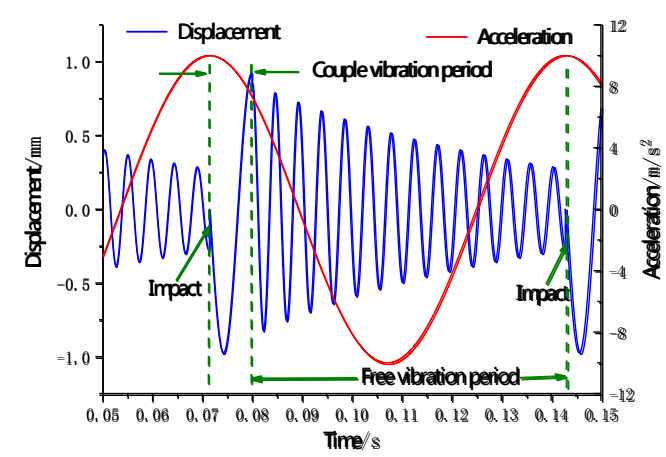

(b) feature in an impact period

Fig. 4 Vibration response curve of the HFB

When the HFB is impacted, it will vibration and deform as shown in Fig.5. Thus the capacitance between counter electrode and electrode will change. While the free end has a deformation of $z_{M}$ under force, the deformation of cantilever at $\tau$ is

$$
\delta(\tau)=\frac{Z_{M}}{2 L} \tau^{2}(3 L-\tau)
$$

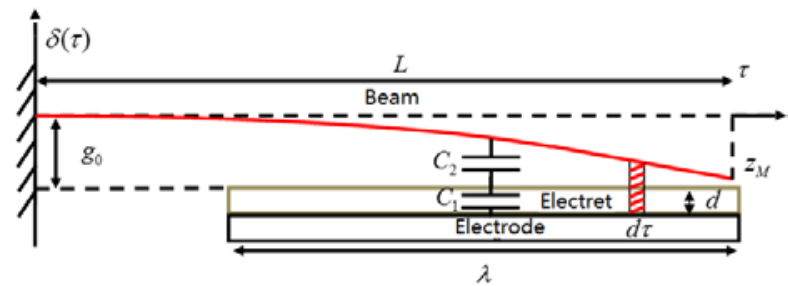

Fig. 5 Deformation of the HFB

Where $L$ the length of HFB is, $\tau$ is the distance from the fix end of HFB. According to the equation (1) and equation (7), the capacitance between counter electrode and electrode is

$$
C(t)=\varepsilon_{0} w \int_{L-\lambda}^{L} \frac{d \tau}{g_{0}-\frac{Z_{M}}{2 L} \tau^{2}(3 L-\tau)+d / \varepsilon}
$$

According to equation (1), (7), (8), the changes of capacitance and load voltage during an impact period can be obtained by Matlab, as shown in Fig. 6, the parameters of calculation are $g_{0}=1.1 \mathrm{~mm}$, $R=10 \mathrm{M} \Omega$, and the surface voltage $-500 \mathrm{~V}$.

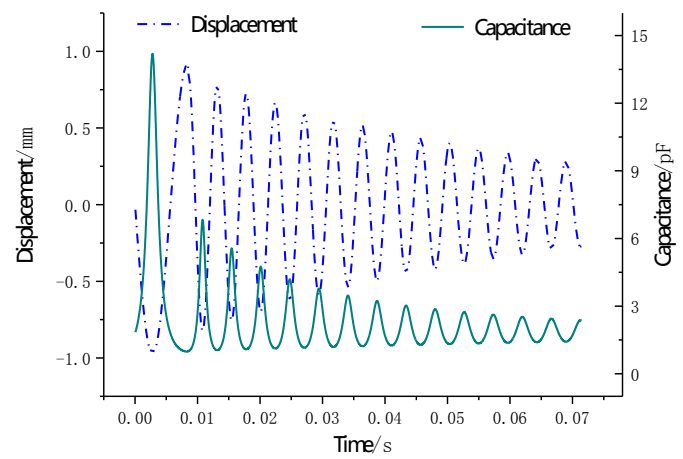

(a) Capacitance change

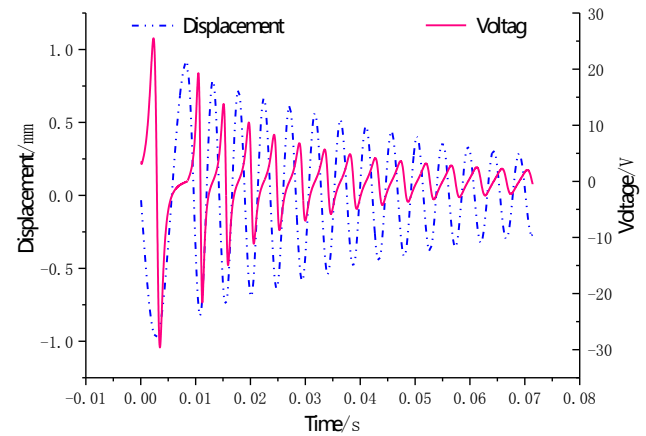

(b) Voltage change

Fig. 6 Change curves of capacitance and voltage during an impact period 
From Fig.6, it can be found that there is no phase difference between capacitance of harvester unit and the displacement of HFB, and the capacitance reaches maximum value when the displacement of HFB reaches maximum. For the output voltage, there is phase difference between the voltage and displacement.

\section{Test and Analyze an Up-conversion E-VEH}

According to the theory model mentioned above, a small-scale prototype of up-conversion E-VEH is designed and fabricated. The LHB and HFB are made of 304 stainless steel, and the HFB is used as counter electrode. The electret film is made of PP with $-500 \mathrm{~V}$ surface potential. The electrode is made of 304 stainless steel, and bonded to the electret by conductive adhesive. The parameters of this E-VEH are list in the Table 1, and the object is shown in Fig.7.

Table 1 Parameters of the E-VEH

\begin{tabular}{cc}
\hline Parameter & Value \\
\hline Volume of LFB & $30 \mathrm{~mm}^{*} 16 \mathrm{~mm}^{*} 0.1 \mathrm{~mm}$ \\
Volume of HFB & $30 \mathrm{~mm}^{*} 16 \mathrm{~mm}^{*} 0.4 \mathrm{~mm}$ \\
Volume of mass & $8 \mathrm{~mm}^{*} 16 \mathrm{~mm} * 10 \mathrm{~mm}$ \\
$E$ of 304 stainless steel & $193 \mathrm{GPa}$ \\
$v$ of 304 stainless steel & 0.3 \\
Density of mass & $7780 \mathrm{~kg} / \mathrm{m}^{3}$ \\
Volume of eletret & $30 \mathrm{~mm} * 16 \mathrm{~mm}^{*} 0.03 \mathrm{~mm}$
\end{tabular}

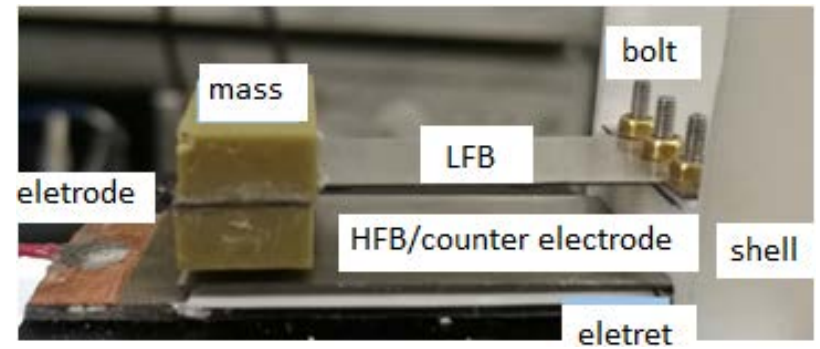

Fig. 7 Photo of the E-VEH prototype

To test performance of the prototype, a measurement system is established as shwon in Fig.8. The prototype is mounted on a small vibrator, which is excited by signal generator and amplifier. The vibration frequency and amplitude can be controlled by the signal generator and the amplifier. An accelerator is mounted on the vibrator to sense the vibration acceleration and recorded by a signal analyzer. The output voltage of the E-VEH prototype is recorded by an oscilloscope.

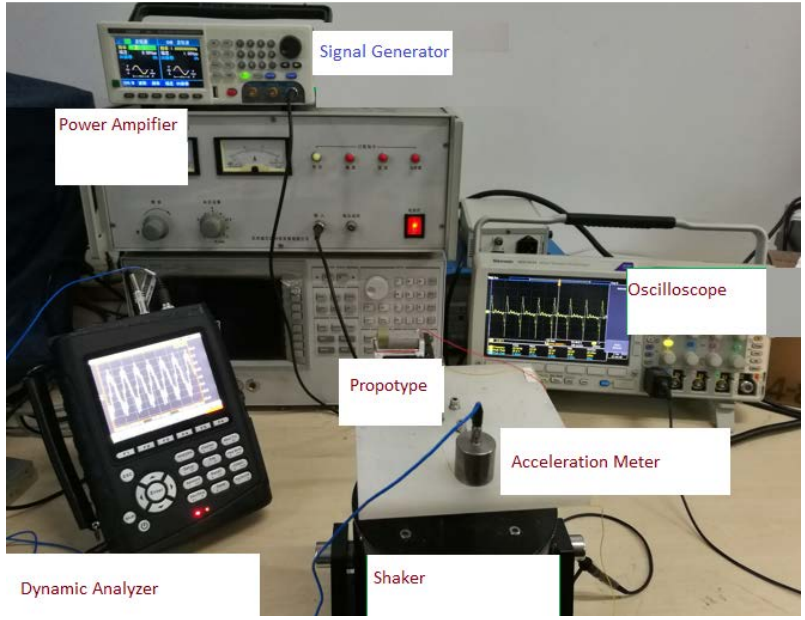

Fig. 8 Photo of the measurement system 
The excitation acceleration is set up $2 \mathrm{~m} / \mathrm{s}^{2}, 5 \mathrm{~m} / \mathrm{s}^{2}$ and $8 \mathrm{~m} / \mathrm{s}^{2}$ separately. For each excitation acceleration, the E-VEH response is observed and recorded by a sweep method. The response curves are shown in Fig.9. According to the measurement results, it can be found that the output voltage reaches maximum at $15 \mathrm{~Hz}$ for each excitation acceleration, and the greater acceleration the greater output. In addition, with the acceleration increasing, there are other output peaks. A peak appears at the point of $29 \mathrm{~Hz}$ for $5 \mathrm{~m} / \mathrm{s}^{2}$ and $8 \mathrm{~m} / \mathrm{s}^{2}$, and another peak appears at the point of $69 \mathrm{~Hz}$ for $8 \mathrm{~m} / \mathrm{s}^{2}$. According to the prototype structure, the first three orders natural frequency of LFB are $8.5 \mathrm{~Hz}$, $29.3 \mathrm{~Hz}, 65.7 \mathrm{~Hz}$. In other words, the harvester can harvest the lower frequency vibration.
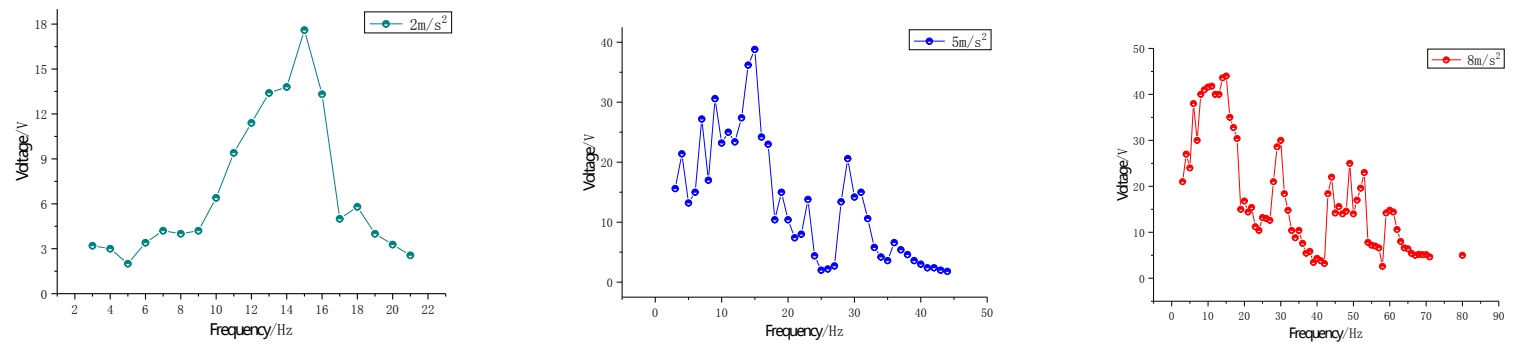

Fig. 9 Curves of the prototype output

\section{Conclusions}

For small-scale E-VEHs, its natural frequency always relative high, while environment vibration frequency is low. This cause the harvest efficiency is relative low. An up-conversion harvester structure is designed and analyzed. By measurement, this structure can convert part of the vibration energy within the vibration frequency between $1 \mathrm{~Hz}$ and $70 \mathrm{~Hz}$ into electrical energy under the action of the excitation load with the amplitude is $8 \mathrm{~m} / \mathrm{s}^{2}$ after the introduction of the up-conversion structure.

\section{References}

[1] Ling, C.S., Dan, H., Steve, G.B. Technological challenges of developing wireless health and us a GE monitoring systems. Proc. SPIE 2013.

[2] Karami, M.A., Inman, D.J. Powering pacemakers from heart beat vibrations using linear and nonlinear energy harvesters. Appl. Phys. Lett. 100 (2012), 042901.

[3] Mitcheson, P.D., Green, T.C., Yeatman, E.M. Power processing circuits for electromagnetic, electrostatic and piezoelectric inertial energy scavengers. Microsyst. Technol. 13(2007) 1629-1635.

[4] Wen Lo, H., Tai, Y.-C. Parylene-based electret power generators. J. Micromech. Microeng. 18 (2008) 104006.

[5] Wang, F., Hansen, O. Invisible surface charge pattern on inorganic electrets. IEEE Electron. Device Lett. 34 (2013) 1047-1049.

[6] Chunhui Gao, Shiqiao Gao, Haipeng Liu, Lei Jin and Junhu Lu. Electret Length Optimization of Output Power for Double-End Fixed Beam Out-of-Plane Electret-Based Vibration Energy Harvesters. Energies, 10 (2017) 1122

[7] Chunhui Gao, Shiqiao Gao, Haipeng Liu, Lei Jin, Junhu Lu, Ping L. Optimization for output power and band width in out-of-plane vibration energy harvesters employing electrets theoretically, numerically and experimentally. Microsystem Technology, 23 (2017) 5759-5769

[8] Zhang, Y.; Luo, A.; Xu, Y.; Wang, T.; Zhang, A.; Wang, F. Electret-based electrostatic energy harvesting device with the MEMS technology. In Proceedings of the 2016 12th IEEE/ASME 
International Conference on Mechatronic and Embedded Systems and Applications (MESA), Auckland, New Zealand, 29-31 August 2016. 